\title{
Raman scattering and anti-Stokes emission from a single spherical microcavity with a CdTe quantum dot monolayer
}

\author{
Yu. P. Rakovich and J. F. Donegan ${ }^{\text {a) }}$ \\ Semiconductor Photonics Group, Department of Physics, Trinity College, Dublin 2, Ireland \\ N. Gaponik \\ Institute of Physical Chemistry, University of Hamburg, 20146 Hamburg, Germany
}

\author{
A. L. Rogach \\ Photonics and Optoelectronics Group, Physics Department and CeNS, University of Munich, \\ 80799 Munich, Germany
}

(Received 23 April 2003; accepted 3 August 2003)

\begin{abstract}
We have studied the Raman and luminescence spectra of a microcavity-quantum dot system consisting of a melamine formaldehyde latex microsphere coated by CdTe colloidal quantum dots. The cavity-induced enhancement of the Raman scattering allows the observation of Raman spectra from only a monolayer of CdTe quantum dots. Periodic structure with very narrow peaks in the luminescence spectra of a single microsphere was detected arising from the coupling between the emission from quantum dots and spherical cavity modes. Strong anti-Stokes emission from CdTe quantum dots coupled to the cavity modes was observed using low intensity below band-gap excitation and attributed to the strong field enhancement at the microcavity resonances. (C) 2003 American Institute of Physics. [DOI: 10.1063/1.1615316]
\end{abstract}

The unique optical properties of spherical microcavities have attracted a lot of interest. ${ }^{1}$ Whispering gallery mode (WGM) oscillations within a single microsphere doped by semiconductor nanocrystals [colloidal quantum dots (QDs)] have been the subject of theoretical and experimental studies for the last three years. ${ }^{2-6}$ The combination of the highquality factor $(Q)$ and the small mode volume of glass microspheres with tunable emission properties of QDs has made it possible to observe narrow resonant structure in emission spectra, ${ }^{2}$ to detect the modification of photoluminescence (PL) decay lifetimes ${ }^{5,6}$ and lasing. ${ }^{3,6}$ Recently, we have developed a core-shell system consisting of a melamine formaldehyde (MF) latex microsphere coated by CdTe QDs. The high optical transparency and thermal and mechanical stability of MF make it ideal as a candidate in optical applications while CdTe QDs produce emission in the spectral region from $500-750 \mathrm{~nm}$.

CdTe QDs capped with thioglycolic acid were synthesized in aqueous medium as described elsewhere. ${ }^{7}$ The colloidal solution of CdTe QDs with a PL maximum at $620 \mathrm{~nm}$ (2.4 $\mathrm{nm}$ radius) and a PL quantum efficiency of $\sim 25 \%$ at room temperature was used for coating MF microspheres with a monolayer of QDs utilizing the layer-by-layer deposition technique. ${ }^{8}$ The diameter of the MF spheres used was 2.2 microns, which is much smaller than that employed in previous studies $^{2-6}$ allowing us to provide the optimal excitation conditions like matching the laser wavelength to one of the WGM frequencies and achieving good correlation between the WGM and the laser linewidths. ${ }^{1}$ We proceed from the assumption that enhancement of PL efficiency as well as Raman scattering can result from cavity quantum electrody-

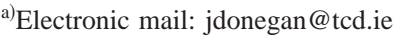

namic effects because of a very small mode volume in our samples.

Absorption and PL spectra of aqueous solutions of colloidal QDs were measured using Shimadzu-3101 and Spex Fluorolog spectrometers, respectively. The Raman spectra and PL spectra from a single microsphere were recorded in a backscattering geometry using a Renishaw micro-Raman system $\left(1800 \mathrm{~mm}^{-1}\right.$ grating, $>1 \mathrm{~cm}^{-1}$ resolution). The spatial resolution of less than $1 \mu \mathrm{m}$ was provided using a microscope with a $100 \times$ objective lens. For all measurements, the microspheres were deposited on a Si wafer. Raman spectra were excited by a $488 \mathrm{~nm}$ line of an $\mathrm{Ar}^{+}$laser with a power of $1.8 \mathrm{~mW}$. An $\mathrm{Ar}^{+}$laser (wavelength $\lambda=514.5 \mathrm{~nm}$, $1.5 \mathrm{~mW}$ power) or $\mathrm{He}-\mathrm{Ne}$ laser $(\lambda=632.8 \mathrm{~nm}$, power up to $20 \mathrm{~mW}$ ) were used in the micro-PL measurements.

The optical spectra of colloidal CdTe QDs in water are presented in Fig. 1, demonstrating the excellent optical quality by the pronounced peak in absorption and a single band edge PL band. The blueshift of the QDs absorption band by $\sim 610 \mathrm{meV}$ with respect to bulk CdTe indicates a strong electronic quantum confinement effect.

The measured Raman spectrum of a single MF/CdTe microsphere with a shell thickness of only one monolayer reveals three pronounced features as shown in Fig. 2. The use of the $\mathrm{Si}$ substrate provides the built-in standard of the $\mathrm{Si}$ transverse optical (TO) mode at $520 \mathrm{~cm}^{-1}$. The peak at $162.0 \mathrm{~cm}^{-1}$ originates from the longitudinal optical (LO) phonon mode of the CdTe QD monolayer. The frequency of this peak is smaller than the corresponding bulk value of 168 $\mathrm{cm}^{-1}$ (Ref. 9) by $6.0 \mathrm{~cm}^{-1}$. This shift originates from two sources: a redshift due to confinement of optical phonons, ${ }^{10}$ and a blueshift caused by lattice contraction. ${ }^{11}$ The 25.0 $\mathrm{cm}^{-1}$ width of the LO phonon line for CdTe QDs in our experiment reflects the somewhat broad size distribution of 


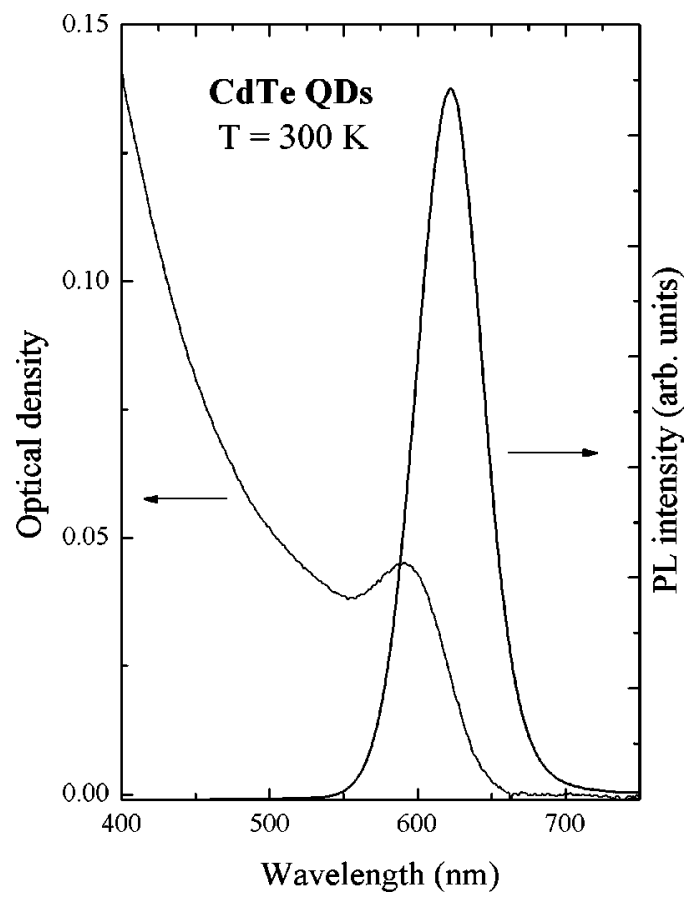

FIG. 1. Room-temperature absorption and PL spectra of the CdTe QDs in aqueous solution.

the quantum dots (about 12\% as estimated from the spectral width of the absorption peak). The narrow peak at 975.5 $\mathrm{cm}^{-1}$ (full width at half maximum $=16.5 \mathrm{~cm}^{-1}$ ) is assigned to the melamine ring vibrations. ${ }^{12}$ It can be seen that the intensity of the Raman peak from the single monolayer of CdTe QDs is comparable to the signal from MF proving that resonance Raman spectroscopy allows very efficient vibrational characterization of just a monolayer of semiconductor QDs coating the microsphere. Such an enhancement of the Raman efficiency can be explained by the high optical feedback provided by the WGM resonances of the microsphere. ${ }^{1}$

In contrast to the broad, featureless PL band in the spectra of colloidal QDs (Fig. 1), the emission spectra of a single $\mathrm{MF} / \mathrm{CdTe}$ microsphere exhibit a very sharp periodic structure (Fig. 3), which is superimposed on a broad background signal. Note that the spectra in Fig. 3 are presented without

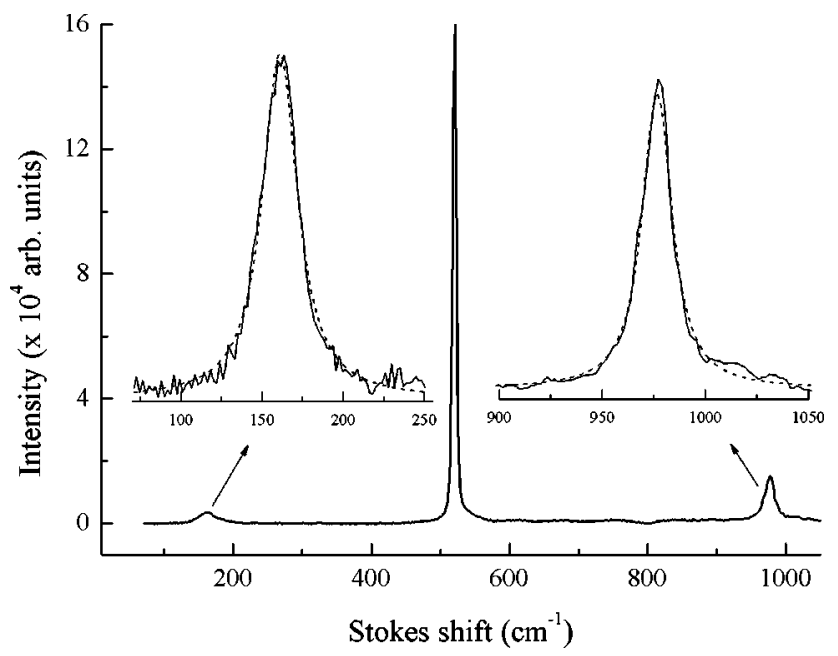

FIG. 2. The Raman spectrum of a single MF/CdTe microsphere on Si sub-

strate. Excitation by $\mathrm{Ar}^{+}$laser $(\lambda=488 \mathrm{~nm})$.
Downloaded $24 \mathrm{Sep} 2003$ to 134.226.1.114. Redistribution subject to AIP license or copyright, see http://ojps.aip.org/aplo/aplcr.jsp

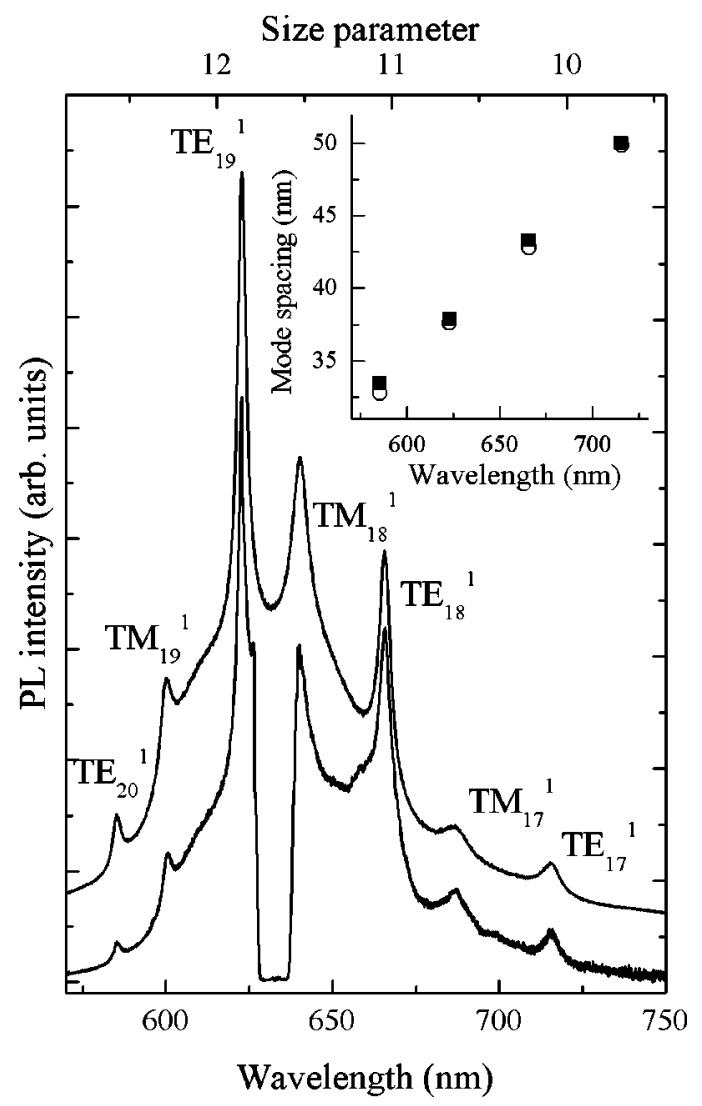

FIG. 3. Room-temperature PL spectra from a single MF microsphere coated by one monolayer of CdTe QDs excited by $\mathrm{Ar}^{+}$laser (above band-gap excitation, $\lambda=514 \mathrm{~nm}$, upper curve) and $\mathrm{He}-\mathrm{Ne}$ laser (below band-gap excitation, $\lambda=632.8 \mathrm{~nm}$, lower curve). The anomalous decrease of the PL intensity in the wavelength region from 626 to $640 \mathrm{~nm}$ is due to the notch filter used. Inset: Experimental (circles) and calculated (squares) spacing between adjacent TE modes.

background subtraction of the broad emission from the QDs. The sharp peaks can be ascribed to the transverse electrical (TE) and transverse magnetic (TM) resonant cavity modes with a different angular quantum number $n$ and a radial quantum number $\ell{ }^{1}$ The polarization character of the modes in spherical microcavities with QDs has been identified using polarization micro-PL measurements with different a orientation of the analyzer axis and excitation polarization. The results of the mode identification $\left(\mathrm{TE}_{n}^{\ell}\right.$ or $\left.\mathrm{TM}_{n}^{\ell}\right)$ are shown in Fig. 3.

In the spectral region of low absorption $(625-750 \mathrm{~nm})$, the intensity of the TE modes is smaller than that of the TM modes and the TE peaks are narrower in comparison with TM peaks of the same $n$ in agreement with theoretical predictions. ${ }^{1}$ As a result, the quality factor $Q=\omega_{\text {cav }} / \Delta \omega_{\text {cav }}$ of the TE modes is found to be almost twice as high as that of TM modes. For a given $\ell$, the quality factor increases with the angular quantum number, ranging from $Q=67$ for $\mathrm{TE}_{17}^{1}$ peak to $Q=177$ for $\mathrm{TE}_{19}^{1}$. The quality factor of such very small microspheres is limited by different loss mechanisms among which the most important are the leakage of light due to high radius of curvature and surface inhomogeneities. The accuracy of the mode assignment can be verified comparing the experimental and calculated spectral spacing between adjacent modes. For the WGM peaks shown in Fig. 3, the value of the angular quantum number is much higher than the size parameter $x=\pi d / \lambda$ throughout the whole spectral region $(d$ 
is the diameter of the microsphere). In this case, the approximate distance between two resonances of successive modes with the same order and polarization can be obtained from the expression: ${ }^{13}$

$$
\Delta \lambda_{n}=\frac{\lambda_{n}^{2} \tan ^{-1}\left\{n_{r}-1\right\}^{1 / 2}}{\pi d\left\{n_{r}-1\right\}^{1 / 2}},
$$

where $\lambda_{n}$ is the emission wavelength of the WGM peaks with different $n$ and $n_{r}$ is the refractive index of the sphere (1.68). According to Eq. (1), the mode spacing decreases with increasing mode number $n$. The calculated and experimentally observed values for $\Delta \lambda$ are shown in the inset of Fig. 3 demonstrating excellent agreement and indicating that the monolayer of semiconductor material with a high refractive index does not influence the dielectric constant of the microcavity. The spectral position and spacing of WGM peaks depend only on the size of the microcavity and are insensitive to the variation of the excitation wavelength in the spectral region above the absorption peak.

We also studied the optical behavior of the microcavityquantum dot system with excitation below the band gap of the CdTe QDs in the region of low absorption. In addition to the normal Stokes-shifted luminescence, we make the observation of anti-Stokes emission. The tail of anti-Stokes PL (ASPL) can be seen ranging up to $\sim 315 \mathrm{meV}$ above the excitation energy (Fig. 3, lower curve). The ASPL process is certainly highly efficient having an intensity comparable to the Stokes PL as seen from Fig. 3. We found that the integrated intensity of ASPL has an almost linear dependence on the excitation intensity under weak or moderate excitation $\left(<200 \mathrm{~W} / \mathrm{cm}^{2}\right)$. This dependence is very similar to the behavior of ASPL in colloidal CdTe QDs where the progressive transition from Stokes PL into ASPL can be observed when changing the excitation wavelength to below the band-gap region. ${ }^{14}$ Based on the linear dependence of the ASPL intensity, we propose multiphonon-assisted excitation of an electron from the ground state to the excited state through the mediation of the shallow trap levels as a mechanism of ASPL in our samples. This mechanism is more likely to be the cause of photon energy upconversion in our case, because (i) high quantum efficiency of Stokes PL in CdTe QDs is indicative of passivated deep trap levels, and (ii) thermal excitation (or re-excitation) of the trapped carriers cannot be eliminated in our experiments which were carried out at room temperature. In the case of colloidal QDs such a low cross-section mechanism, like anti-Stokes excitation can be only efficient in samples with high enough quantum yields. ${ }^{14}$ The observation of ASPL from a CdTe/MF microsphere can once again be attributed to the optical feedback via the microcavity with a WGM structure which leads to an increased probability of energy transfer to the emitting species. ${ }^{1}$ As a result, strong coupling between photonic states of spherical microcavity and electronic states of CdTe QDs can be achieved simultaneously in both Stokes and anti-Stokes spectral regions (Fig. $3)$.

In conclusion, Raman spectra from a monolayer of CdTe quantum dots were observed due to strong coupling with the microcavity. Simultaneous Stokes and anti-Stokes emission were realized by low intensity excitation below the band gap. Microspheres coated by chemically synthesized CdTe QDs should find use for photonic applications as a resonant system with well controllable wavelengths of the emission peaks.

The financial support for this work was provided by the Science Foundation Ireland (Grant No. 02/IN.1/I47) and the DFG program "Photonic Crystals". The authors thank Dr. A. Moore and Dr. T. Perova for help with experimental arrangements.

${ }^{1}$ Optical Processes in Microcavities, edited by R. K. Chang and A. J. Chamillo (World Scientific, Singapore, 1996).

${ }^{2}$ M. Artemyev and U. Woggon, Appl. Phys. Lett. 76, 1353 (2000).

${ }^{3}$ V. I. Klimov and M. G. Bawendi, MRS Bull. 26, 998 (2001).

${ }^{4}$ X. Fan, P. Palinginis, S. Lacey, and H. Wang, Opt. Lett. 25, 1600 (2000).

${ }^{5}$ X. D. Fan, M. C. Lonergan, Y. Z. Zhang, and H. L. Wang, Phys. Rev. B 64, 115310 (2001).

${ }^{6}$ M. V. Artemyev, U. Woggon, R. Wannemacher, H. Jaschinski, and W. Langbein, Nano Lett. 1, 309 (2001).

${ }^{7}$ N. Gaponik, D. V. Talapin, A. L. Rogach, K. Hoppe, E. V. Shevchenko, A. Kornowski, A. Eychmüller, and H. Weller, J. Phys. Chem. B 106, 7177 (2002).

${ }^{8}$ A. S. Susha, F. Caruso, A. L. Rogach, G. B. Sukhorukov, A. Kornowski, H. Möhwald, M. Giersig, A. Eychmüller, and H. Weller, Colloids Surf., A 163, 39 (2000).

${ }^{9}$ M. Gorska and W. Nazarewicz, Phys. Status Solidi B 65, 193 (1974).

${ }^{10} \mathrm{C}$. Trallero-Giner, A. Debernardi, M. Cordona, and E. Menendez-Proupin, Phys. Rev. B 57, 4664 (1998).

${ }^{11}$ J.-Yu. Zhang, X.-Y. Wang, M. Xiao, L. Qu, and X. Peng, Appl. Phys. Lett. 81, 2076 (2002)

${ }^{12}$ M. L. Scheepers, R. J. Meier, L. Markwort, J. M. Gelan, D. J. Vanderzande, and B. J. Kip, Vib. Spectrosc. 9, 139 (1995).

${ }^{13}$ P. Chylek, J. Opt. Soc. Am. A 7, 1609 (1990).

${ }^{14}$ Yu. P. Rakovich, S. A. Filonovich, M. J. M. Gomes, J. F. Donegan, D. V. Talapin, A. L. Rogach, and A. Eychmüller, Phys. Status Solidi B 229, 449 (2002). 